Annales Mathematicae Silesianae 31 (2017), 63-70

DOI: $10.1515 /$ amsil-2016-0020

\title{
OUTER MEASURES ON A COMMUTATIVE RING INDUCED BY MEASURES ON ITS SPECTRUM
}

\author{
Dariusz Dudzik, Marcin SkrzyŃSKI*
}

\begin{abstract}
On a commutative ring $R$ we study outer measures induced by measures on $\operatorname{Spec}(R)$. The focus is on examples of such outer measures and on subsets of $R$ that satisfy the Carathéodory condition.
\end{abstract}

\section{Preliminaries and introduction}

Throughout the note, $R$ stands for a nonzero commutative ring with identity and $R^{\times}$denotes the set of invertible elements of $R$. We define $\operatorname{Spec}(R)$ to be the spectrum of $R$, i.e., the family of all prime ideals $\wp \subset R$. The family of all maximal ideals of $R$ will be denoted by $\operatorname{Max}(R)$. Recall that $\operatorname{Max}(R) \subseteq \operatorname{Spec}(R)$ and

$$
\bigcup \operatorname{Spec}(R)=R \backslash R^{\times}=\bigcup \operatorname{Max}(R) .
$$

By "measure" we always mean a "non-negative $\sigma$-additive measure". The power set of a set $X$ is denoted by $2^{X}$. We use the following definition of an outer measure.

Received: 03.08.2016. Accepted: 18.11.2016. Published online: 07.02.201\%.

(2010) Mathematics Subject Classification: 13A15, 28A12.

Key words and phrases: outer measure, commutative ring, prime ideal, Carathéodory's condition, ring of functions, unique factorization domain.

${ }^{*}$ Corresponding author. 
Definition 1.1. A function $\varphi: 2^{X} \rightarrow[0,+\infty]$ is said to be an outer measure on a set $X$, if it satisfies two conditions:

(i) $\varphi(A) \leqslant \sum_{n=1}^{\infty} \varphi\left(B_{n}\right)$ for every sequence $\left\{B_{n}\right\}_{n=1}^{\infty}$ of subsets of $X$ and every set $A$ such that $A \subseteq \bigcup_{n=1}^{\infty} B_{n}$,

(ii) $\varphi(\emptyset)=0$.

We refer to [1] for more information about commutative rings and to [3, 4] for elements of measure theory.

Consider a family $\mathcal{P} \subseteq \operatorname{Spec}(R)$ such that $\bigcup \mathcal{P}=R \backslash R^{\times}$. Consider also a $\sigma$-algebra $\mathfrak{M}$ of subsets of $\mathcal{P}$. Let $\mu: \mathfrak{M} \rightarrow[0,+\infty]$ be a measure. Given any set $A \subseteq R$, we define

$$
\Omega(A)=\left\{\mathcal{S} \in \mathfrak{M}: \bigcup \mathcal{S} \supseteq A \backslash R^{\times}\right\} .
$$

In [2] we proved that

$$
\mu^{*}: 2^{R} \ni A \mapsto \inf _{\mathcal{S} \in \Omega(A)} \mu(\mathcal{S}) \in[0,+\infty]
$$

is an outer measure on the ring $R$. This outer measure will be referred to as the outer measure induced by $\mu$. The main theorem of [2] shows that $\mu^{*}$ behaves well with respect to elementwise multiplication of sets.

The present note is a continuation of [2]. Our purpose is twofold: to characterize subsets of $R$ that satisfy the Carathéodory condition with respect to $\mu^{*}$ and to discuss some quite general examples of the outer measures induced by measures on spectra.

\section{Some measures on spectra and the outer measures induced by them}

For any element $a \in R$, we define $(a)$ to be the principal ideal of the ring $R$ generated by $a$. Suppose that $R$ is a unique factorization domain and is not a field. Let $E$ be the set of all irreducible elements of $R$. Notice that, by the definition of an irreducible element, $E \subseteq R \backslash\left(R^{\times} \cup\{0\}\right)$. Moreover, since $R$ is not a field, we have $E \neq \emptyset$. Let us define $\mathcal{P}_{\text {irr }}(R)=\{(a): a \in E\}$. Then $\mathcal{P}_{\text {irr }}(R) \subseteq \operatorname{Spec}(R)$ and 


$$
\bigcup \mathcal{P}_{\text {irr }}(R)=R \backslash R^{\times}
$$

(because $R$ is a unique factorization domain).

Consider now a nonempty set $F \subseteq E$ and the map $\Phi: F \ni a \mapsto(a) \in$ $\mathcal{P}_{\text {irr }}(R)$. If $\mathfrak{N}$ is a $\sigma$-algebra of subsets of $F$ and $\nu: \mathfrak{N} \rightarrow[0,+\infty]$ is a measure, then $\mathfrak{M}=\left\{\mathcal{S} \subseteq \mathcal{P}_{\text {irr }}(R): \Phi^{-1}(\mathcal{S}) \in \mathfrak{N}\right\}$ is a $\sigma$-algebra of subsets of $\mathcal{P}_{\text {irr }}(R)$ and

$$
\mu: \mathfrak{M} \ni \mathcal{S} \mapsto \nu\left(\Phi^{-1}(\mathcal{S})\right) \in[0,+\infty]
$$

is a measure. Let us take a closer look on the outer measure $\mu^{*}: 2^{R} \rightarrow[0,+\infty]$.

Proposition 2.1. In the situation described above, assume additionally that the map $\Phi$ is bijective. Then, for any $A \subseteq R$, we have $\mu^{*}(A)=\inf _{G \in \widetilde{\mathfrak{N}}} \nu(G)$, where

$$
\widetilde{\mathfrak{N}}=\left\{G \in \mathfrak{N} \mid \forall x \in A \backslash R^{\times} \exists g \in G: g \text { is a divisor of } x\right\} .
$$

Proof. By the surjectivity of $\Phi$ and the definition of a principal ideal, a set $\mathcal{S} \in \mathfrak{M}$ belongs to $\Omega(A)$ if and only if

$$
\forall x \in A \backslash R^{\times} \exists g \in \Phi^{-1}(\mathcal{S}): g \text { is a divisor of } x .
$$

Pick an arbitrary $G \subseteq F$. The injectivity of $\Phi$ yields that $\Phi^{-1}(\Phi(G))=G$, and hence $\Phi(G) \in \mathfrak{M}$ if and only if $G \in \mathfrak{N}$. We thus obtain

$$
\begin{aligned}
& \{\mu(\mathcal{S}): \mathcal{S} \in \Omega(A)\} \\
& =\left\{\nu\left(\Phi^{-1}(\mathcal{S})\right) \mid \mathcal{S} \in \mathfrak{M}, \forall x \in A \backslash R^{\times} \exists g \in \Phi^{-1}(\mathcal{S}): g \text { is a divisor of } x\right\} \\
& =\left\{\nu(G) \mid G \in \mathfrak{N}, \forall x \in A \backslash R^{\times} \exists g \in G: g \text { is a divisor of } x\right\} \\
& \quad=\{\nu(G): G \in \widetilde{\mathfrak{N}}\} .
\end{aligned}
$$

In view of the definition of $\mu^{*}$, the proof is complete.

In fact, the bijectivity assumption above means that the set $F$ contains precisely one element from each class of associate elements of the set $E$.

ExAmple 2.2. Let $R=\mathbb{Z}$, the ring of integers, $F=\mathbb{P}$, the set of prime numbers, and $\nu$ be the counting measure on $\mathbb{P}$. Observe that

$$
\mathcal{P}_{\text {irr }}(\mathbb{Z})=\{(p): p \in \mathbb{P}\}=\operatorname{Max}(\mathbb{Z}) .
$$


Therefore, $\Phi: \mathbb{P} \ni p \mapsto(p) \in \mathcal{P}_{\text {irr }}(\mathbb{Z})$ is a bijection and the measure $\mu$ coincides with the counting measure on $\mathcal{P}_{\text {irr }}(\mathbb{Z})$.

Consider next the set $A=\{-14,-5,-1,0,6,9,15,28\}$. Define

$$
\widetilde{\mathfrak{N}}=\left\{G \subseteq \mathbb{P} \mid \forall x \in A \backslash \mathbb{Z}^{\times} \exists g \in G: g \text { is a divisor of } x\right\}
$$

and recall that $\mathbb{Z}^{\times}=\{-1,1\}$. Since $3 \in \mathbb{P}, 5 \in \mathbb{P}$ and $9=3^{2}$, we get that $\{3,5\} \subseteq G$ for any $G \in \widetilde{\mathfrak{N}}$. However, neither 3 nor 5 is a divisor of 28 , and hence $\{3,5\} \notin \widetilde{\mathfrak{N}}$. It is evident that $\{2,3,5\} \in \widetilde{\mathfrak{N}}$ and $\{3,5,7\} \in \widetilde{\mathfrak{N}}$. By Proposition 2.1 , we obtain

$$
\mu^{*}(A)=\inf _{G \in \tilde{\mathfrak{N}}} \nu(G)=\nu(\{2,3,5\})=3 .
$$

Let us turn to function rings. Consider a nonempty set $X$ and a field $\mathbb{F}$. We denote by $\mathbb{F}^{X}$ the ring of all functions $f: X \rightarrow \mathbb{F}$ (pointwise operations). Let $R$ be a subring of $\mathbb{F}^{X}$ such that every constant function belongs to $R$ (in other words, $\mathbb{F} \subseteq R$ ) and $R^{\times}=\{f \in R: f(x) \neq 0$ for all $x \in X\}$. If $x \in X$, then $\wp_{x}=\{f \in R: f(x)=0\}$ is a maximal ideal of the ring $R$. Notice also that

$$
\bigcup_{x \in X} \wp_{x}=R \backslash R^{\times}
$$

We now define $\mathcal{P}_{X}(R)=\left\{\wp_{x}: x \in X\right\}$. Consider the map $\Psi: X \ni x \mapsto$ $\wp_{x} \in \mathcal{P}_{X}(R)$. If $\mathfrak{N}$ is a $\sigma$-algebra of subsets of $X$ and $\nu: \mathfrak{N} \rightarrow[0,+\infty]$ is a measure, then identically to the previous part of the section, $\mathfrak{M}=\{\mathcal{S} \subseteq$ $\left.\mathcal{P}_{X}(R): \Psi^{-1}(\mathcal{S}) \in \mathfrak{N}\right\}$ is a $\sigma$-algebra of subsets of $\mathcal{P}_{X}(R)$ and

$$
\mu: \mathfrak{M} \ni \mathcal{S} \mapsto \nu\left(\Psi^{-1}(\mathcal{S})\right) \in[0,+\infty]
$$

is a measure.

Proposition 2.3. In the situation described above, assume additionally that $\Psi$ is an injection. Then, for any $A \subseteq R$, we have $\mu^{*}(A)=\inf _{Y \in \mathfrak{N}_{0}} \nu(Y)$, where

$$
\mathfrak{N}_{0}=\left\{Y \in \mathfrak{N} \mid \forall f \in A \backslash R^{\times}: Y \cap f^{-1}(0) \neq \emptyset\right\} .
$$

Proof. The proof is completely analogous to the proof of Proposition 2.1 The crucial point is that a set $\mathcal{S} \in \mathfrak{M}$ belongs to $\Omega(A)$ if and only if

$$
\forall f \in A \backslash R^{\times} \exists x \in \Psi^{-1}(\mathcal{S}): f(x)=0 .
$$


The injectivity of $\Psi$ means that $R$ separates the points in the set $X$. Hence, if $X$ is a normal topological space and $\mathbb{F} \in\{\mathbb{R}, \mathbb{C}\}$, then $\mathcal{C}(X, \mathbb{F})$, the ring of all continuous functions $f: X \rightarrow \mathbb{F}$, satisfies the assumptions of Proposition 2.3 . If $n$ is a positive integer and $\mathbb{F}$ is an algebraically closed field, then so does the polynomial ring $\mathbb{F}\left[x_{1}, \ldots, x_{n}\right]$. Proposition 2.3 can also be applied to various rings of differentiable or holomorphic functions. The proposition generalizes [2, Proposition 3].

Let us finally discuss an example showing that $\mathcal{P}_{X}(R)$ does not have to coincide with $\operatorname{Max}(R)$.

ExAmple 2.4. Suppose that $X$ is an infinite set. Define $I$ to be the family of all functions $f: X \rightarrow \mathbb{F}$ with the property that

$$
\exists Y \subseteq X:\left\{\begin{array}{l}
Y \text { is finite, } \\
f(x)=0 \text { for any } x \in X \backslash Y .
\end{array}\right.
$$

Then $I$ is a proper ideal of the $\operatorname{ring} R=\mathbb{F}^{X}$. Let $Z$ be an infinite subset of $X$ such that $X \backslash Z$ is also infinite. Consider the function $h \in R$ defined by

$$
h(x)= \begin{cases}1, & \text { if } x \in Z, \\ 0, & \text { if } x \notin Z .\end{cases}
$$

Since $h \notin I$ and $I+R h$ is a proper ideal of $R$, we get $I \notin \operatorname{Max}(R)$. However, obviously, $I \subseteq \wp$ for some $\wp \in \operatorname{Max}(R)$. Observe that

$$
\forall x \in X \exists f \in I: f(x) \neq 0 .
$$

Consequently, no point $x \in X$ has the property that $f(x)=0$ for all $f \in \wp$. This yields $\wp \notin \mathcal{P}_{X}(R)$.

\section{3. $\mu^{*}$-measurable sets}

We begin with a very brief recapitulation of the Carathéodory condition. Let $\varphi$ be an outer measure on a set $X$.

Definition 3.1. A set $A \subseteq X$ is said to be $\varphi$-measurable (or to satisfy the Carathéodory condition with respect to $\varphi$ ), if

$$
\forall T \subseteq X: \varphi(T)=\varphi(T \cap A)+\varphi(T \backslash A) .
$$


Theorem 3.2 (Carathéodory). The totality $\mathfrak{M}$ of $\varphi$-measurable subsets of $X$ is a $\sigma$-algebra. Moreover,

(i) the restriction $\left.\varphi\right|_{\mathfrak{M}}$ is a measure,

(ii) if $\varphi(A)=0$ for some $A \subseteq X$, then $A \in \mathfrak{M}$.

It is obvious that $\mathfrak{M} \supseteq\left\{A \in 2^{X}: \varphi(X \backslash A)=0\right\}$. The class of "obviously $\varphi$-measurable" sets is, in fact, a bit larger. We will say that a set $A \subseteq X$ satisfies condition $(\bullet)$ with respect to $\varphi$, if $\varphi(B) \in\{0,+\infty\}$ for any $B \subseteq A$.

Proposition 3.3. Let $A \subseteq X$. Suppose that either $A$ or $X \backslash A$ satisfies condition $(\bullet)$ with respect to $\varphi$. Then $A$ is a $\varphi$-measurable set.

Proof. Pick an arbitrary $T \subseteq X$. By the definition and the monotonicity of an outer measure,

$$
\max \{\varphi(T \cap A), \varphi(T \backslash A)\} \leqslant \varphi(T) \leqslant \varphi(T \cap A)+\varphi(T \backslash A) .
$$

Hence, $\varphi(T)=\varphi(T \backslash A)$ whenever $\varphi(T \cap A)=0$, and $\varphi(T)=\varphi(T \cap A)$ whenever $\varphi(T \backslash A)=0$. It is obvious that $\varphi(T)=+\infty$ whenever $\varphi(T \cap A)=$ $+\infty$ or $\varphi(T \backslash A)=+\infty$. Condition $(\bullet)$ therefore implies that $\varphi(T)=\varphi(T \cap$ $A)+\varphi(T \backslash A)$.

Let us also recall some properties of the outer measure $\mu^{*}: 2^{R} \rightarrow[0,+\infty]$ induced by a measure $\mu$ on a suitable set $\mathcal{P} \subseteq \operatorname{Spec}(R)$.

Proposition 3.4. If $A, B \subseteq R \backslash R^{\times}$and $C \subseteq R$, then

(i) $\mu^{*}(A B)=\min \left\{\mu^{*}(A), \mu^{*}(B)\right\}$,

(ii) $\mu^{*}(C)=\mu^{*}\left(C \backslash R^{\times}\right)$.

The above proposition is a part of [2, Theorem 1]. Notice that the set $C$ is $\mu^{*}$-measurable whenever $C \subseteq R^{\times}$or $C \supseteq R \backslash R^{\times}$.

We are now ready to state and prove the main result of the note.

Theorem 3.5. Let $A \subseteq R \backslash R^{\times}$be such that $0<\mu^{*}(A)<+\infty$. Assume moreover that $0<\mu^{*}(B)<+\infty$ for some $B \subseteq R \backslash\left(A \cup R^{\times}\right)$. Then the set $A$ is not $\mu^{*}$-measurable.

Proof. Suppose, in order to derive a contradiction, that $A$ is $\mu^{*}$-measurable. Pick arbitrary sets $E \subseteq A$ and $Z \subseteq R$. Define $T=E Z \cup A$. Then

$$
\mu^{*}(T)=\mu^{*}(T \cap A)+\mu^{*}(T \backslash A)=\mu^{*}(A)+\mu^{*}(E Z \backslash A) .
$$


If $\mathcal{S} \in \Omega(A), a \in E$ and $b \in Z$, then $a \in \wp$ for some $\wp \in \mathcal{S}$ (because $E \subseteq A$ and $\left.A \subseteq R \backslash R^{\times}\right)$, and hence $a b \in \wp$. This proves that $\Omega(A) \subseteq \Omega(E Z)$. Consequently, $\Omega(A) \subseteq \Omega(E Z \cup A)=\Omega(T)$. We therefore obtain that

$$
\mu^{*}(A) \geqslant \mu^{*}(T)=\mu^{*}(A)+\mu^{*}(E Z \backslash A) .
$$

Since $\mu^{*}(A)<+\infty$, the above inequality yields $\mu^{*}(E Z \backslash A)=0$. Thus we have proved the following property:

$$
\forall E \subseteq A \forall Z \subseteq R: \mu^{*}(E Z \backslash A)=0 .
$$

Define now $W=A B \cup B$. Recall that $B \cap A=\emptyset$. Combining the property we have just proved with the $\mu^{*}$-measurability of $A$, we get

$$
\mu^{*}(A B)=\mu^{*}(A B \cap A)+\mu^{*}(A B \backslash A)=\mu^{*}(A B \cap A) .
$$

Consequently,

$$
\mu^{*}(W \cap A)=\mu^{*}((A B \cap A) \cup(B \cap A))=\mu^{*}(A B \cap A)=\mu^{*}(A B) .
$$

Notice also that by the monotonicity of $\mu^{*}$,

$$
\mu^{*}(W \backslash A)=\mu^{*}((A B \backslash A) \cup(B \backslash A)) \geqslant \mu^{*}(B \backslash A)=\mu^{*}(B) .
$$

The same argument as in the previous part of the proof shows that $\Omega(B) \subseteq$ $\Omega(W)$, and hence $\mu^{*}(B) \geqslant \mu^{*}(W)$. It follows therefore from the $\mu^{*}$-measurability of $A$ that

$$
\mu^{*}(B) \geqslant \mu^{*}(W)=\mu^{*}(W \cap A)+\mu^{*}(W \backslash A) \geqslant \mu^{*}(A B)+\mu^{*}(B) .
$$

Since $\mu^{*}(B)<+\infty$, the above inequalities yield $\mu^{*}(A B)=0$. But by Proposition 3.4 (i) we have $\mu^{*}(A B)=\min \left\{\mu^{*}(A), \mu^{*}(B)\right\}>0$, a contradiction.

It seems worth noting that a set $A \subseteq R$ is $\mu^{*}$-measurable if and only if so is $A \backslash R^{\times}$(this follows from the fact that every subset of $R^{\times}$is $\mu^{*}$-measurable). Recall also that $A$ is $\mu^{*}$-measurable if and only if so is $R \backslash A$. In view of these two equivalences and Proposition 3.4 (ii), our main theorem implies the following corollary.

Corollary 3.6. If $A \subseteq R$ is a $\mu^{*}$-measurable set and neither $A$ nor $R \backslash A$ satisfies condition $(\bullet)$ with respect to $\mu^{*}$, then $\mu^{*}(A)=+\infty=\mu^{*}(R \backslash A)$.

As an easy consequence of the main theorem, we also obtain a complete characterization of $\mu^{*}$-measurable sets in the case where $\mu^{*}(R)<+\infty$. 
COROLlary 3.7. Suppose that $\mu^{*}$ is finite (i.e., $\mu^{*}(R)<+\infty$ ). Then $A \subseteq R$ is a $\mu^{*}$-measurable set if and only if either $\mu^{*}(A)=0$ or $\mu^{*}(R \backslash A)=0$.

Let us conclude the note with an example concerning the case where $\mu^{*}(R)=+\infty$.

EXAmple 3.8. Consider the measure $\mu: 2^{\operatorname{Max}(\mathbb{Z})} \rightarrow[0,+\infty]$ defined by

$$
\mu(\{(p)\})= \begin{cases}+\infty, & \text { if } p=2, \\ 1 / p, & \text { if } p \in \mathbb{P} \backslash\{2\} .\end{cases}
$$

We can apply Proposition 2.1 to the outer measure $\mu^{*}: 2^{\mathbb{Z}} \rightarrow[0,+\infty]$. If $k \in \mathbb{Z} \backslash\{-1,0,1\}$ is neither a power of 2 nor the opposite of a power of 2 , then $\mu^{*}(\{k\})=1 / p_{k}$, where $p_{k}$ stands for the largest prime divisor of $k$. Notice also that $\mu^{*}(\{0\})=0$. Therefore, a set $A \in 2^{\mathbb{Z}}$ satisfies condition (•) with respect to $\mu^{*}$ if and only if

$$
A \backslash\{-1,0,1\} \subseteq \bigcup_{n=1}^{\infty}\left\{-2^{n}, 2^{n}\right\} .
$$

Observe now that $\mu^{*}(\mathbb{P})=+\infty=\mu^{*}(\mathbb{Z} \backslash \mathbb{P})$. It is obvious that $\mathbb{P}$ and $\mathbb{Z} \backslash \mathbb{P}$ do not satisfy condition $(\bullet)$ with respect to $\mu^{*}$. Define $T=\{3,9\}$. Then

$$
\mu^{*}(T)=\mu^{*}(T \cap \mathbb{P})=\mu^{*}(T \backslash \mathbb{P})=1 / 3 .
$$

Consequently, $\mathbb{P}$ is not a $\mu^{*}$-measurable set.

\section{References}

[1] Atiyah M.F., MacDonald I.G., Introduction to Commutative Algebra, Addison-Wesley Publishing Company, Reading, 1969.

[2] Dudzik D., Skrzyński M., An outer measure on a commutative ring, Algebra Discrete Math. 21 (2016), no. 1, 51-58.

[3] Federer H., Geometric Measure Theory, Springer, Berlin, 1969.

[4] Halmos P.R., Measure Theory, Springer, New York, 1976.

Institute of Mathematics

Pedagogical University of Cracow

PODCHORĄŻYCH 2

30-084 KRAKÓW

POLAND

e-mail: dariusz.dudzik@gmail.com
Institute of Mathematics

Cracow University of Technology

WARSZAWSKA 24

31-155 KRAKÓW

POLAND

e-mail: pfskrzyn@cyf-kr.edu.pl 